\title{
A prática da psicologia escolar e sua contribuição na inclusão escolar de crianças
}

\section{atípicas}

\author{
The practice of school psychology and its contribution in school inclusion of atypical children \\ La práctica de la psicología escolar y su contribución en la inclusión escolar de niños atípicos
}

Recebido: 17/03/2021 | Revisado: 24/03/2021 | Aceito: 01/04/2021 | Publicado: 11/04/2021

Pedro Wilson Ramos da Conceição

ORCID: https://orcid.org/0000-0002-3868-4917 Pontifícia Universidade Católica de Minas Gerais, Brasil

E-mail: Pedro_wilson_ramos@hotmail.com

Jacqueline da Silva Santos

ORCID: https://orcid.og/0000-0001-9240-3867

Centro Universitário Maurício de Nassau, Brasil

E-mail: Jack.10silva@hotmail.com

Gilciane Silva Magalhães

ORCID: https://orcid.org/0000-0003-1272-1369

Centro Universitário Maurício de Nassau, Brasil

E-mail: Gilcianemagalhaes157@gmail.com

Wadenia Sousa de Carvalho

ORCID: https://orcid.org/0000-0002-8067-7542

Centro Universitário Maurício de Nassau, Brasil

E-mail: Urdenia2323@gmail.com

Bianca Maria Silva Alves

ORCID: https://orcid.org/0000-0002-8032-4843

Centro Universitário Maurício de Nassau, Brasil

E-mail: ancabia48@gmail.com

Aparecido Minino dos Santos

ORCID: https://orcid.org/0000-0002-3520-9950

Faculdade Montenegro, Brasil

E-mail: Aparecidosantos1111@hotmail.com

Luciana Almeida Mota

ORCID: https://orcid.org/0000-0001-5449-4139

Centro Universitário Cesumar, Brasil

E-mail: lucianamt@gmail.com

Kelvisson Rodrigues de Carvalho

ORCID: https://orcid.org/0000-0002-1088-0094

Centro Universitário Maurício de Nassau, Brasil

E-mail: kelvissonrodriggues@gmail.com

Lucas Costa Ferreira

ORCID: https://orcid.org/0000-0002-3001-023X

Centro Universitário Maurício de Nassau, Brasil

E-mail: lucasmag27@hotmail.com

Zaira Arthemisa Mesquita Araujo

ORCID: https://orcid.org/0000-0002-6625-034X

Pontifícia Universidade Católica de Minas Gerais, Brasil

E-mail: Zaira.psicologia@hotmail.com

Érika Castelo Branco Said

ORCID: https://orcid.org/0000-0003-1152-3424

Centro Integrado de Reabilitação do Piauí, Brasil

E-mail: erikacbsaid@hotmail.com

\section{Resumo}

O presente trabalho objetivou compreender a atuação do psicólogo escolar na inclusão de crianças atípicas. Os dados foram coletados mediante entrevista realizada com uma psicóloga da Associação de Amigos dos Autistas do Piauí (AMA). As respostas da entrevista foram categorizadas em três categorias: a concepção de desenvolvimento atípico e de inclusão escolar, a atribuição da psicologia escolar e a contribuição do psicólogo escolar para a inclusão escolar de crianças atípicas. Os resultados mostraram que pode se entender o desenvolvimento atípico como um padrão de desenvolvimento que não é considerado normativo para a faixa etária do indivíduo, fazendo-se necessário um projeto de inclusão bem elaborado na instituição de ensino. Cabendo ao psicólogo escolar trabalhar em conjunto com a rede escolar, os familiares dos alunos e a comunidade, oferecendo amparo e orientação, visando tornar a escola um ambiente proporcionador de desenvolvimento igualitário.

Palavras-chave: Desenvolvimento atípico; Psicologia escolar; Educação inclusiva. 


\begin{abstract}
This study aimed to understand the role of the school psychologist in the inclusion of atypical children. Data were collected through a seeding interview conducted with a psychologist from the Association of Friends of Autistic people of Piauí (AMA). The answers of the interview were categorized into three categories: the conception of atypical development and school inclusion, the attribution of school psychology and the contribution of the school psychologist to the school inclusion of atypical children. The results showed that atypical development can be understood as a development pattern that is not considered normative for the age group of the individual, making it necessary to have a well-elaborated inclusion project in the educational institution. It is up to the school psychologist to work together with the school network, the students' families and the community, offering support and guidance, aiming to make the school an environment that provides for equal development.
\end{abstract}

Keywords: Atypical development; School psychology; Inclusive education.

\title{
Resumen
}

Este estudio tenía como objetivo comprender el papel del psicólogo escolar en la inclusión de niños atípicos. Los datos fueron recogidos a través de una entrevista realizada con un psicólogo de la Asociación de Amigos del pueblo autista de Piauí (AMA). Las respuestas de la entrevista se clasificaron en tres categorías: la concepción del desarrollo atípico y la inclusión escolar, la atribución de la psicología escolar y la contribución del psicólogo escolar a la inclusión escolar de los niños atípicos. Los resultados mostraron que el desarrollo atípico puede entenderse como un patrón de desarrollo que no se considera normativo para el grupo de edad del individuo, por lo que es necesario tener un proyecto de inclusión bien elaborado en la institución educativa. Corresponde al psicólogo escolar trabajar junto con la red escolar, las familias de los estudiantes y la comunidad, ofreciendo apoyo y orientación, con el objetivo de hacer de la escuela un entorno que proporcione el mismo desarrollo.

Palabras clave: Desarrollo atípico; Psicología escolar; Educación inclusiva.

\section{Introdução}

O desenvolvimento atípico caracteriza-se por um comportamento tido anormal a sua faixa etária, oriundo de transtornos de aprendizagem e transtornos intelectuais ou de ambiente desfavorável ao seu desenvolvimento (Abreu, 2006). Crianças com esse tipo de desenvolvimento apresentam dificuldades de aprendizagens e de socialização, requerendo uma maior atenção e estimulação de sua aprendizagem. Atualmente a educação de crianças atípicas se faz mediante uma perspectiva de escolarização regular inclusiva.

Diante dessa perspectiva, a inclusão escolar se faz mediante uma modificação pedagógica que inclua todos os educandos, para atender o ritmo de aprendizagem de cada um. Além da elaboração de estratégias de adaptação de recurso, técnicas e avaliações didáticas que contribuem para a aprendizagem de todos, sejam atípico ou não, ademais deve-se leva em consideração o aperfeiçoamento dos profissionais envolvidos (Mantoa, 2000).

Infelizmente a descriminação e preconceito no âmbito social e educacional ainda são comuns, cabendo ao psicólogo e a rede escolar a responsabilidade de modificar essa situação. O processo inclusivo ocorre com a adequação do processo ensinoaprendizagem da escola a essas demandas. Cabendo ao psicólogo escolar analisar os processos de ensino e aprendizagem, utilizando seu conhecimento cientifico acerca do desenvolvimento emocional, cognitivo e social. Visando a educação para todos, por meio de um trabalho em conjunto como a família e a rede escolar, para reduzir os efeitos negativos que dificultam o processo de educação dos educandos.

Diante dessa discussão, pretendeu-se com o presente artigo, compreender a atuação do psicólogo escolar na inclusão de crianças atípicas. Com o intuito de contribuir para uma maior compreensão acerca da atuação do psicólogo escolar com crianças atípicas no âmbito escolar, tendo em vista que o assunto tratado é de extrema importância para o saber cientifico e acadêmico. E com isso, possibilitar à sociedade, um amplo esclarecimento sobre a psicologia escolar, sua contribuição na inclusão de crianças atípicas e como ocorre o processo de inclusão, informando também os desafios enfrentados nesse processo. 


\section{Metodologia}

A presente pesquisa é destinada a acadêmicos e profissionais de psicologia, visando o conhecimento cientifico acerca da contribuição da psicologia escolar para a inclusão de crianças atípicas no ensino regular. A pesquisa foi construída inicialmente por uma análise bibliográfica acerca da atuação do psicólogo escolar, das concepções de desenvolvimento atípico e inclusão escolar e por fim a contribuição do saber e da prática do psicólogo escolar na inclusão de crianças com desenvolvimento atípico no contexto escolar. Posteriormente foi realizada uma pesquisa de campo de carácter qualitativo, analisando e interpretando os assuntos subjetivos do comportamento humano em sua forma mais profunda e complexa (Lara \& Molina, 2015). De acordo com Yin (2010), a entrevista qualitativa não se prende a um roteiro rígido, devendo ocorrer de forma conversacional, onde as perguntas feitas aos participantes poderão sofrer alterações de acordo com o ambiente e o contexto em que a entrevista é realizada. Sendo assim, as perguntas a serem feitas devem ser abertas, para não se limitar as respostas dadas pelos entrevistados.

Foram coletados dados mediante entrevista semeestrutura e gravada com um profissional de psicologia escolar, sendo o mesmo devidamente informados sobre o estudo e seus direitos na pesquisa de acordo com a resolução 466/12 do Ministério da Saúde (Brasil, 1996) e a resolução nº 510/2016 sobre a ética em pesquisas em ciências humanas e sociais. A entrevista é um dos principais instrumentos de coleta de dados utilizados em pesquisa, consistindo em um instrumento importante para o estudo cientifico. Segundo Lüdke e André (1986), a entrevista permite uma captura imediata das informações almejadas. A entrevista deste estudo foi gravada em áudio com auxílio de um gravador, e foi concedida formalmente pelo participante através do Termo de Consentimento Livre e Esclarecido (TCLE), sendo transcrita na íntegra (incluindo aspectos subjacentes à entrevista, como silêncios, pausas, etc.). O participante assinou o Termo de Consentimento Livre e Esclarecido (TCLE), entregue em duas vias tendo ficado uma com o sujeito e outra com o pesquisador. Com o consentimento do sujeito, foi utilizado um gravador durante a entrevista, com a finalidade de preservar o conteúdo original dos relatos, além de facilitar a compreensão do que foi expresso. O roteiro da entrevista semiestruturada foi composto por quinze perguntas, capturadas por gravação de voz para melhor armazenamento dos dados colhidos, que foram transcritos na pesquisa tal qual for respondido pelo entrevistando. A entrevista encontra-se no Apêndice.

Os dados coletados na entrevista foram analisados mediante a metodologia de análise de conteúdo por categorização, que segundo Carlomagno e Rocha (2016) trata-se uma classificação e categorização dos conteúdos obtidos, reduzindo-os a elementos fundamentais que sejam compatíveis com outros elementos. Fornecendo dessa forma a descrição precisa dos conteúdos comunicativos.

\section{Resultados e Discussões}

No capítulo a seguir será abordado e discutido o resultado obtido com a análise do material coletado por meio da entrevista semiestruturada.

O psicólogo da Associação de Amigos dos Autistas do Piauí (AMA) que participou da pesquisa foi analisado de acordo com os dados obtidos na entrevista semiestruturada. Sendo composta por um profissional de psicologia que atua na Associação de Amigos dos Autistas do Piauí (AMA) de Teresina-Piauí, foi identificado o sexo, idade, estado de formação, anos de profissão, tempo de atuação na área de especialização. O participante foi representado pela inicial A, respeitando os aspectos éticos descritos na metodologia.

No que diz respeito aos dados obtidos do participante, nota-se que a amostra é composta por um participante do sexo feminino, de 28 anos, brasileira e formada pela Universidade Estadual do Piauí (UESPI) a 6 anos; é especialista em Neuropsicologia e Análise do Comportamento. Atua com crianças atípicas a 6 anos. 
Posteriormente foram elaboradas categorias (embasadas no referencial teórico) relacionadas com os temas identificados na entrevista, e que contemplassem os objetivos do trabalho. As discussões levantadas foram embasadas e referenciadas a partir da literatura disponível sobre o desenvolvimento atípico e a atuação do psicólogo escolar na inclusão dessas crianças. Com isso, os dados obtidos a partir da entrevista foram categorizados em: a concepção de desenvolvimento atípico e de inclusão escolar, a atribuição da psicologia escolar e a contribuição do psicólogo escolar para a inclusão escolar de crianças atípicas.

\subsection{A atribuição da psicologia escolar}

O psicólogo escolar realiza um trabalho em conjunto não apenas com a rede escolar, é necessário que também que busque o apoio dos familiares dos alunos e da comunidade. Suas funções vão desde orientar a escola a escolher um conteúdo programático que vai favorecer todos os alunos, como também é trabalho do psicólogo proporcionar a promoção de saúde e bem-estar na instituição de ensino, devendo auxiliar a escola a se adequar aos alunos com algum tipo de deficiência, que de acordo com o participante A depende muito das demandas de cada criança:

[...] Vai das demandas que temos, tudo é feito com as demandas que aparecem, posso fazer algumas solicitações das demandas principais, por exemplo, cadeirante é algo que toda escola sabe que precisa fazer mudança, mas se ela não faz com relação os espaços, de estruturas entre corredores, se cabe a cadeira, até rampas, corrimão, isso a gente auxiliar [...] (Psicóloga A, 2020).

O profissional de psicologia deve auxiliar a escola a tonar-se um ambiente inclusivo, que proporcione um desenvolvimento pleno de alunos típico e atípicos. Proporcionando uma convivência pautada num ambiente que inclua o indivíduo, adequando-o a um convívio igualitário. Alunos que apresentam desenvolvimento atípico, demonstram déficits comportamentais que afetam os seus relacionamentos sociais (Gibela, Reis \& Gioia, 2009). Para se trabalhar esse aspecto comportamental, de acordo com a participante A, é necessário um trabalho em conjunto com todos os alunos e não apenas com aquele que demonstra dificuldades.

[...] Com relação a interação quando esta é falha e deficitária, a gente nunca trabalha a criança sozinha, é preciso trabalhar toda a turma, para que eles estejam preparados para isso, e que os colegas possam dar um modelo adequado para essa outra criança [...] (Psicóloga A, 2020).

Indivíduos atípicos podem ter baixa tolerância a frustração e com isso apresentam um comportamento agressivo e problemático, sendo agressivo com professores e colegas em determinadas situações, ocasionando seu afastamento dos demais alunos, originando um ambiente que não é propicio para o seu desenvolvimento. (LOPES, 2016) De acordo com o participante A, esse aspecto do comportamento do indivíduo deve ser tratado de forma correta para não ocasionar a piora de seu comportamento.

[...] Então o que a gente faz, recebeu um não, é realmente momento de receber aquele não, se perdeu em nenhum momento posso dizer que isso não aconteceu, a gente vai trabalhar é com a regulação dela nesse choro. Se até a atenção for reforçadora para a criança, não importa o que estou falando, se for dada atenção para ela naquele momento de birra aquilo já é reforçador, então tenho que tomar cuidado. Vou ter que avaliar o caso, fazer uma análise funcional desse caso, para saber o que está sendo reforçador para ela continuar com as crises de birra, com a tolerância baixa a frustração, e o que pode fazer com que essa frequência diminua. Vou trabalhar também com toda a sala, toda a comunidade escolar se possível, para trabalhar questões do tipo, por exemplo, em jogos, dar modelos adequados para essa criança de como perder, de como ganhar [...] (Psicóloga A, 2020). 


\subsection{A concepção de desenvolvimento atípico e de inclusão escolar}

O desenvolvimento atípico refere-se a um atraso e/ou prejuízo significativo no desenvolvimento do indivíduo em relação a sua faixa etária. Acarretando dificuldades físicas, cognitivas e psicossociais. O nível de dificuldade depende muito do nível de comprometimento da criança e da estimulação recebida. Segundo Lopes (2016) o desenvolvimento atípico caracterizase por um comprometimento biológico, relacionado a um ambiente desfavorável ou por uma deficiência física e/ou cognitiva do indivíduo. Para a participante A, a concepção de desenvolvimento atípico é considerado:

[...] tudo aquilo que foge do padrão o que é considerado normativo para o desenvolvimento, então, quando a gente estuda desenvolvimento infantil, o juvenil e o adulto, vemos aqueles marcos que precisam acontecer no desenvolvimento físico, cognitivo, psicossocial do indivíduo, tudo aquilo que é fora disso, fora desse padrão normativo é considerado atípico [...] (Psicóloga A, 2020).

O maior desafio das crianças atípicas é romper com as visões preconceituosas e a exclusão escolar e social. Para tanto faz-se necessário o entendimento de que essas crianças precisão do conviver social e escolar igualitário com seus pares para que ocorra uma troca de saberes e experiência. Para isso é de suma importância a inclusão dessas crianças no contexto escolar, visto que a escola é a porta de entrada para a socialização. Diante disso faz-se necessário pontuar sobre o que é a inclusão escolar. Segundo o participante A, a inclusão vai além de um único contexto.

[...] ela envolve, primeiramente todas as áreas, não apenas a questão escolar em si, e ela envolve não apenas o deficiente, mas toda a diversidade que possa existir, ela basicamente seria de fato você não apenas integrar o indivíduo, colocar um indivíduo dentro de uma escola, mas sim dar todas as possibilidades para que ele possa acompanhar tudo que tá acontecendo de maneira plena, então incluir seria você oferecer o desenvolvimento pleno para esse indivíduo, que é considerado atípico ou que é considerado diferente [...] (Psicóloga A, 2020).

Portanto incluir é oferecer um desenvolvimento completo a todo e qualquer indivíduo independente de sua condição física, cognitiva, social, financeira e racial/étnica, oferecendo-os as mesmas oportunidades de desenvolvimento e aprendizagem. Fazendo-se adaptações que facilitem o desenvolvimento conjunto.

[...] A nossa legislação, as nossas leis falam que nós devemos oferecer para os indivíduos o desenvolvimento pleno, o nosso trabalho enquanto sociedade, enquanto comunidade escolar deve ser você oferecer esse desenvolvimento pleno, social, cognitivo. E para o indivíduo que é considerado atípico deve ser a mesma coisa, então incluir é você dar as mesmas oportunidades que você dá para os outros. E para isso você vai ter que fazer toda uma adaptação, mas é você dar essas mesmas oportunidades, é você oferecer o que você puder para que esse indivíduo possa ter o desenvolvimento máximo possível [...]. (Psicóloga A, 2020)

Segundo o Art. 205 da Constituição Federal (1988) é direito do Estado e da família em conjunto com visa o pleno desenvolvimento da pessoas para a cidadania e para o trabalho. Cabe, portanto a escola e a sociedade a compreensão de que são eles que devem se adaptar e se adequar da melhor maneira possível a essas crianças e não o contrário.

\subsection{A contribuição da psicologia escolar na inclusão de crianças atípicas}

$\mathrm{O}$ trabalho do psicólogo escolar em relação às crianças atípicas está voltado para o aspecto de incluir em definitivo o aluno no processo de ensino, gerando mudanças de perspectiva das pessoas que fazem parte desse processo, em relação ao indivíduo atípico. De acordo com a psicóloga $\mathrm{A}$, o quanto mais cedo esse indivíduo começa o processo de interação escolar, melhor seria. 
[...] Enquanto psicóloga escolar e psicóloga que estuda o desenvolvimento, acredito que o mais cedo possível, se as crianças hoje entram com 3 anos, então coloca com 3 anos, se tivéssemos um processo de inclusão que acontecesse como deveria acontecer ia ser o máximo, pois essa criança ia ser estimulada em todos os níveis dela, a tendência é que ela melhorasse muito, inclusive o prognostico clinico dela seria melhor, mas como não temos inclusão de fato, vemos que não é o que acontece [...] (Psicóloga A, 2020).

Com o início da inclusão de alunos atípicos, é essencial que ocorra uma readaptação do processo de ensino da instituição, se adequando aos diferentes tipos de necessidades especiais dos alunos. Essas mudanças devem contribuir para o processo de aprendizagem do aluno em sala, tendo em vista as modificações que esse processo ocasiona na perspectiva educacional (Souza, 2017). É função do psicólogo escolar, participar desse processo, oferecendo amparo ao estudante que está sendo incluído e orientando os demais alunos, professores e todos os profissionais da instituição nesse processo de acolhimento. Por conseguinte, de acordo com a psicóloga A:

[...] O trabalho do psicólogo na inclusão vai desde de observação desse indivíduo dentro e sala de aula, na estrutura da escola como um todo, até se relacionar com todos os profissionais que estão envolvidos com aquela criança, também se relacionar com a família da criança, se a criança tem o diagnóstico ou não, tem que ser averiguado. Porque muitas vezes a escola precisa de um documento que declare que aquela criança realmente precisa de um acompanhamento mais especializado ou de uma adaptação curricular, geralmente ela quer esse diagnóstico de um profissional de fora $[\ldots]$.

Mas para a psicóloga A, a inclusão ainda não conseguiu ser implantada de forma correta, pois ainda é complicado para as escolas compreenderem o que realmente significa a inclusão de fato, apresentando falhas no processo de incluir os alunos.

[...] E a concepção de inclusão ou a inclusão realmente, ainda hoje algumas escolas não conseguem entender. Então vai desde a parte de não conseguir compreender a inclusão que já é a base, o que você precisa é saber entender o que é a inclusão como ela acontece, o que eu devo fazer para ela acontecer, se eu não entendo sobre isso a minha prática vai ser bem falha, então muitas escolas ou a grande parte delas, não consegue ainda compreender de fato essa inclusão, como ela deve acontecer, ou se compreende não cumpri. Então infelizmente não temos uma inclusão que considera verdadeira, muitas escolas, a maioria das escolas não [...] (Psicóloga A, 2020).

No processo de inclusão o psicólogo precisa fazer acontecer um trabalho em conjunto entre a escola e a família do aluno, pois essa parceria ocasiona uma melhoria no processo de aprendizagem de alunos atípicos. Assim sendo, de acordo com o psicóloga A:

[...] Se percebe que a criança está com um desenvolvimento fora do padrão considerado normativo, então a família é chamada, mas as vezes ela mesmo vem sem ter necessidade de ser chamada e ai a gente vai realmente colocar o que está acontecendo e pedir a parceria dessa família, parceria no sentido dessa família ser colaborativa em falar o que está acontecendo com a criança, ser colaborativa com os terapeutas de fora que vão estar estimulando essa criança, se a escola passa atividade para essa criança fazer em casa essa família precisa estar estimulando, acompanhando [...].

O processo de educação inclusiva busca responder as necessidades dos alunos que fazem parte do processo de inclusão educacional, abrangendo os diferentes ritmos e estilos de aprendizagem, com o intuito de proporcionar uma educação igualitária e de qualidade para todos (Souza, 2017). O psicólogo escolar tem uma contribuição essencial nesse processo, pois é ele que preparam os profissionais para lidarem com essas demandas, oferece apoio as famílias e suporte os discentes. Não olha apenas para o lado biológico dos indivíduos atípicos, pois mesmo com limitações, presenta também as suas potencialidades (Ramos et al., 2016). 


\section{Considerações Finais}

Este trabalho propôs, como objetivo geral, compreender a atuação do psicólogo escolar na inclusão de crianças atípicas. Foram descritas a atribuição da psicologia escolar; apresentada a concepção de desenvolvimento atípico e de inclusão e a contribuição da psicologia escolar na inclusão de crianças atípicas, objetivo presente na fala da entrevistada e na literatura da área. Sendo discutida a atuação do psicólogo escolar frente às demandas atípicas, afirmando que os objetivo mencionados foram atingidos.

A partir dos dados obtidos na entrevista observou-se como resultado que incluir vai além da resolução dos problemas no contexto escolar, trata-se de um trabalho conjunto com toda escola, a família, a comunidade e a criança, isto é, trabalhar com o contexto escolar, familiar e social das crianças, visando seu desenvolvimento.

No entanto apesar da grande conquista da inclusão atual, ainda há muito a se fazer. Notou-se na fala da entrevistada que muitas escolas não incluem de fato a criança, apenas a inserem no contexto escolar. Muitas escolas ainda não possuem psicólogos escolares, como por exemplo, as públicas e algumas limitam as ações do psicólogo.

No tocante a atribuição do psicólogo escolar, notou-se sua grande importância nesse contexto, visto que o mesmo utiliza seu conhecimento teórico-cientifico para adaptações da escola as demandas. Várias são as atribuições do psicólogo dentro da escola, sempre visando à melhoria das disponibilidades de educação para todos. O psicólogo escolar vai além do diagnóstico das demandas, ele investiga o agente causador da problemática, trabalhando a minimização ou eliminação desse agente.

Quanto à concepção do desenvolvimento atípico e de inclusão, percebeu-se que ambas as concepções se interligam, visto que a primeira se trata do "diferente", tido "anormal" pela maioria e a inclusão da igualdade de oportunidades para todos independente de qualquer diferença, seja ela física, biológica, cognitiva, religiosa, racial-étnica ou sexual. Sendo assim, ao se falar sobre crianças atípicas é quase impossível não se abordar sua inclusão social e educacional.

Ao discorrer sobre a contribuição da psicologia escolar na inclusão de crianças atípicas, nota-se a suma importância deste fazer na inclusão dessas crianças. Com seu conhecimento teórico acerca do desenvolvimento humano, das psicopatologias e da importância das interações para o desenvolvimento e aprendizagem das crianças, o psicólogo pode avaliar as demandas, suas dificuldades, suas potencialidades, elaborando com a escola a melhor forma de manejo educacional para determinada demanda. Levando sempre em consideração que a criança é mais que sua dificuldade ou deficiência.

Entretanto, para que a inclusão seja completa, ainda se necessita de conscientização do governo em investimento nessa área, em conscientização da população na compreensão do diferente como normal, das escolas no suporte adequado e de qualidade para efetivação da inclusão.

A pesquisa possui relevância em termos metodológicos e prático-técnico, tanto para os profissionais de psicologia, como para acadêmicos de psicologia, para um melhor conhecimento da área abordada.

Os dados obtidos na presente pesquisa, evidenciaram que para se resolver a questão da inclusão de crianças atípicas nas escolas, é preciso ir além do contexto escolar. Portanto, faz-se necessário a implementação de pesquisas que viabilizem uma outra forma de abordagem desta temática, visando o estudo de aspectos distintos da vida da criança, como o social e o familiar, para que assim se possibilitem novas perspectivas sobre a mesma temática.

\section{Referências}

Abreu, M. C. B. F. (2016). Desenvolvimento de conceitos científicos em crianças com deficiência mental (Dissertação de mestrado). Universidade Católica de Brasília - UCB, Brasília, DF, Brasil. https://bdtd.ucb.br:8443/jspui/handle/123456789/1882.

Barbosa, R. M., \& Marinho-araújo, C. M. (2010). Psicologia escolar no Brasil: Considerações e reflexões históricas. Estudos de Psicologia, 27(3), 393-402. https://www.scielo.br/pdf/estpsi/v27n3/11.pdf 
Batista, M. W., \& Enumo, F. S. (2004). Inclusão escolar e deficiência mental: Análise da interação social entre companheiros. Estudos de Psicologia, 9(1), 101-111. https://www.scielo.br/pdf/epsic/v9n1/22386.pdf

Breitenbach, F. V., Honnef, C., \& Costa, F. A. T. (2016). Educação inclusiva: As implicações das traduções e das interpretações de Declaração de Salamanca no Brasil. Ensaio: Avaliação e Políticas em Educação, 24(90), 359-379. https://www.scielo.br/pdf/ensaio/v24n91/1809-4465-ensaio-24-91-0359.pdf. 10.1590/S0104-40362016000200005

Carlomagno, M. C., \& Rocha, L. C. (2016). Como criar e classificar categorias para fazer análise de conteúdo: Uma questão metodológica. Revista Eletrônica de Ciências Políticas, 7(1), 173-188. https://revistas.ufpr.br/politica/article/view/45771/28756

Cassins, A. M. (2007). Manual de Psicologia Escolar - Educacional. Gráfica e Editora Unificada. https://crppr.org.br/wp-content/uploads/2019/05/157.pdf

Gomes, C., \& Souza, V. L. T. (2011). Educação, Psicologia Escolar e Inclusão: Aproximações necessárias. Revista: psicopedagogia, $28(86)$, 185-193. http://pepsic.bvsalud.org/scielo.php?script=sci_arttext\&pid=S0103-84862011000200009

Lara, A. M. B., Molina, A. A. (2011). Pesquisa qualitativa: Apontamentos, conceitos e tipologias. In C. A. A. Toledo, \& M. T. C. Gonzaga (Org s), Metodologia e técnicas de pesquisa nas áreas de ciências humanas. EEduem.

Lei de Diretrizes e Bases n. 9.394, 20 de dezembro de 1996. Regulamenta o sistema educacional. http://portal.mec.gov.br/seesp/arquivos/pdf/lei93 94_ldbn1.pdf

Lopes, A. P. F. A. (2016). Desenvolvimento atípico, acesso a educação de qualidade (Dissertação de mestrado). Universidade de São Paulo - USP, São Carlos, SP, Brasil. http://pepsic.bvsalud.org/pdf/ptp/v11n2/v11n2a12.pdf

Lüdke, M., \& André, M. E. D. A. (Org.). (1986). Pesquisa em educação: Abordagens qualitativas. (P. 33). Pedagógia e universitária.

Mantoan, M. T. E. (Org.). (2003). Inclusão Escolar: O que é? Por que? Como fazer? Moderna.

Matos, D. C. de, \& Matos, P. G. S. de. (2018). Intervenções em psicologia para inclusão escolar de crianças autistas: estudo de caso. Revista Espaço Acadêmico, 18(211), 21-31. http://www.periodicos.uem.br/ojs/index.php/EspacoAcademico/article/view/44949.

Oliveira, C. B. E., \& Marinho-araújo, C. M. (2009). Psicologia escolar: cenários atuais. Estudos e Pesquisas em Psicologia, 9(3), 648-663. http://pepsic.bvsalud.org/pdf/epp/v9n3/v9n3a07.pdf

Pereira, P. C., \& Matsukura, T.S. (2013). Inclusão escolar e educação infantil: um estudo de caso. Educação especial, 26(45), 125-144. https://periodicos.ufsm.br/index.php/educacaoespecial/article/view/5471

Pereira-Silva, N. L., Andrade, J. F. C. M., Crolman, S. R., \& Mejía, C. F. (2017). O papel do psicólogo escolar: concepções de professores e gestores. Psicologia Escolar e Educacional, 21(3), 407-415. https://www.redalyc.org/pdf/2823/282353802007.pdf

Ramos, F. S., Fontes, A. L. D., Cordeiro, M. M. P., \& Júnior, A. C. S. (2016, novembro). Congresso Internacional de Educação Inclusiva - CINTEDI, Campina Grande, Paraiba, Brasil, 2. file:///C:/Users/Aparecido/Downloads/TRABALHO_EV060_MD1_SA12_ID3239_20102016213613.pdf

Ribela, A. C. P., Reis, P. V. N., \& Gioia, P. S. (2009). Procedimento de ensino de interações sociais entre jovens com desenv olvimento atípico e seus pares baseados na análise do comportamento. Psicologia: Teoria e Práticas, 11(2), 161-181. http://pepsic.bvsalud.org/scielo.php?script=sci_arttext\&pid=S151636872009000200012

Santos, G. M., Silva, L. A. P., Pereira, J. L., Lima, A. G. X., \& Neto, F. L. A. (2018). Atuação e práticas na psicologia escolar: revisão sistemática em periódicos. Psicologia Escolar e Educacional, 22(3), 583-591. https://www.scielo.br/pdf/pee/v22n3/2175-3539-pee-22-03-583.pdf

Santos, J. V., \& Gonçalves, C. M. (2016). Psicologia educacional: importância do psicólogo na escola. O portal dos psicólogos, 1-22. https://www.psicologia.pt/artigos/textos/A1045.pdf

Silva, L. B., Paulino, W. M. S., Dias, S. G., Oliveira, F. P., \& Travassos, L. M. M. (2015). A importância da atuação do profissional de psicologia no contexto escolar. Realize Eventos e Editora,

Souza, B. K. S. (2017). Desenvolvimento atípico e inclusão: concepções de estudantes de ciências naturais (Trabalho de Conclu são de Curso). Universidade de Brasília - UB, Brasília, DF, Brasil. https://www.bdm.unb.br/handle/10483/18192

Vygotsky. L. S. (2011). A defectologia e o estudo do desenvolvimento e da educação da criança anormal. Educação e Pequisa, 37(4), 861-870. https://www.scielo.br/scielo.php?pid=S1517-97022011000400012\&script=sci_arttext\&tlng=pt

Yin, R. K. (2016). Pesquisa qualitative do início ao fim. Métodos de coleta de dados (cap. 6, pp. 138-161). Penso. 